\title{
Exceptional Variation on a Common Theme: The Evolution of Crustacean Compound Eyes
}

\author{
Thomas W. Cronin • Megan L. Porter
}

Published online: 20 September 2008

(C) Springer Science + Business Media, LLC 2008

\begin{abstract}
The Crustacea contain an amazing, and often (to humans) bizarre, array of visual designs. This diversity includes many different examples of both simple and compound eyes, each with standard or uniquely crustacean features. In this review, we focus on the anatomical variation, optical principles, and molecular diversity of crustacean compound eyes to illustrate how the complicated structures involved in vision are adapted for particular environments. Using this knowledge as a starting point, and considering what is known of crustacean evolution overall, we present the most recent ideas of how crustacean compound eyes have evolved and show how eyes that are based on fundamentally different optical principles can in fact be derived from each other and thus be closely related through common descent.
\end{abstract}

Keywords Crustacea Compound eyes · Apposition .

Superposition · Evolution · Opsin

\section{Introduction}

The extant Crustacea contain six classes, 42 orders, 849 families, and somewhere over 52,000 species (Martin and Davis 2001). Although they do not make up the most species-rich group within the Arthropoda (the insects, or hexapods, do), the crustaceans exhibit as great a degree of

T. W. Cronin $(\bowtie) \cdot$ M. L. Porter

Department of Biological Sciences,

University of Maryland Baltimore County,

1000 Hilltop Circle,

Baltimore, MD 21250, USA

e-mail: cronin@umbc.edu morphological diversity as is seen in any other animal phylum (Fig. 1). This variability has led to a scientific fascination with understanding crustacean evolution. As a group, the crustaceans have a predilection for marine habitats, although crustacean species can be found in almost every conceivable type of habitat (e.g. coastal, pelagic, benthic, and deep marine; freshwater; terrestrial). The wide range of light environments represented by these diverse habitats has operated together with the overall morphological diversity within the Crustacea to produce a dizzying array of crustacean visual systems, based on eyes ranging from simple pigment cups to compound eye designs not seen in any other animal. In this review, we focus on the evolution of compound eyes.

No discussion of eye evolution is possible without a general understanding of the evolutionary history of the group of interest. The crustaceans pose a particularly difficult challenge in this regard, however, as there is so much morphological diversity that it is difficult to characterize crustaceans with any single set of features, and attempts at understanding their taxonomic groups and phylogenetic relationships based on morphology have been problematic for as long as they have been studied. Furthermore, molecular studies have shown that the Crustacea are not a unified group, but instead form several distinct lineages, some of which are more closely related to the insects than to other well-accepted crustacean lineages (Fig. 1). While we recognize that our understanding of the evolution of Crustacea is far from complete, we believe that a discussion of eye design, from both anatomical and molecular perspectives, illustrates many key evolutionary principles. Within this review, we highlight some of the typical and unusual eye designs within the Crustacea and discuss their evolutionary implications. 

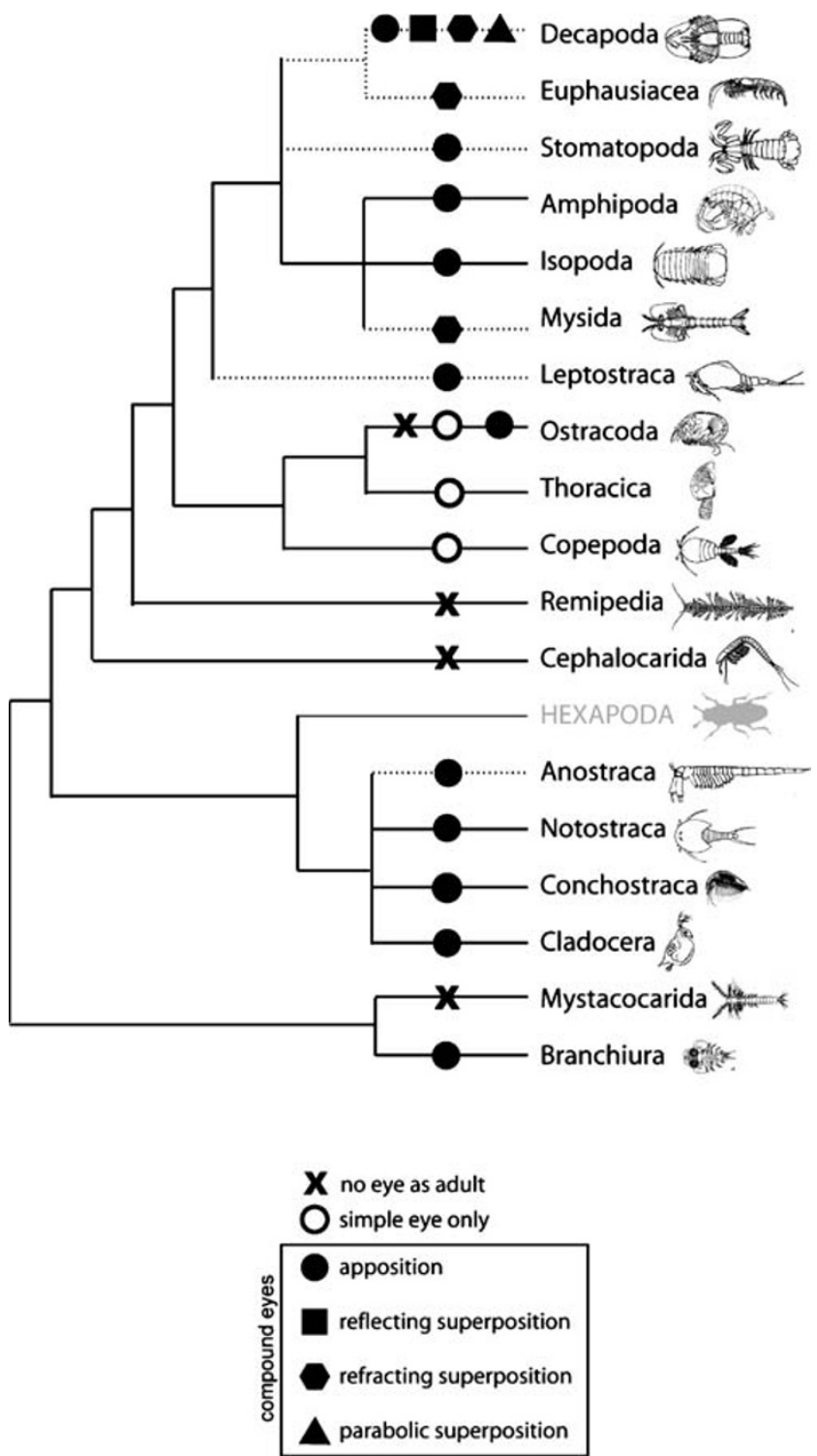

Fig. 1 Phylogenetic distribution of optical eye designs within the major crustacean lineages. Only the eye design of adults is indicated. Dashed branches indicate lineages where compound eyes are on stalks. Topology of the relationships drawn after Schram and Koenemann (2004)

\section{Basics of Compound Eyes}

Almost every optical system known to exist in eyes can be found in one crustacean or another, ranging through a variety of "simple" designs (eyes that have a single aperture and a retinal sheet) to a uniquely diverse set of compound eye types (see Land 1984). While it seems certain that simple eyes arose independently several times in crustacean evolution, little is known about the evolutionary paths taken to produce the very unusual assortment of simple eye designs present in modern crustaceans. Most crustaceans that have only simple eyes are small and unfamiliar as well (see Fig. 1). Consequently, in this review of crustacean visual evolution, we will restrict our discussion to the compound eyes. These are the designs used by all large, well-known crustaceans-lobsters, shrimp, crabs - as well as by a vast diversity of crustaceans that are less familiar to most of us but nevertheless successful and abundant animals in their own right: krill, mysids, water fleas, and mantis shrimps, for example. It is also worth noting that the largest diversity of compound-eye optical designs in any group of animals is found within the larger crustaceans (the malacostracans), particularly within the decapods (Fig. 1).

The oldest eyes in the fossil record are compound eyes; specifically, those of the trilobites. While these are certainly derived from earlier and simpler types that are lost to us, and while trilobites are not thought to be ancestral to any modern arthropods, these eyes illustrate the common design features on which today's compound eyes are based (Fig. 2). The more fundamental trilobite design, called the holochroal type (Clarke 1889), is thought to have operated on the same principles of modern types found in today's crabs, butterflies, and bees, among other animals. Here, each fossilized, preserved facet probably denoted a separate optical unit, called an ommatidium. Ommatidia are the unitary elements of all compound eyes, and in every case, extinct or modern, contain a corneal lens, some optical structures below it, and a group of photoreceptors which typically function as a unit, sampling one point in space. Trilobite holochroal eyes have optics very much like those of modern, nocturnal arthropods (Fordyce and Cronin 1993), suggesting that these animals lived in dimly lit waters or were active only at night.

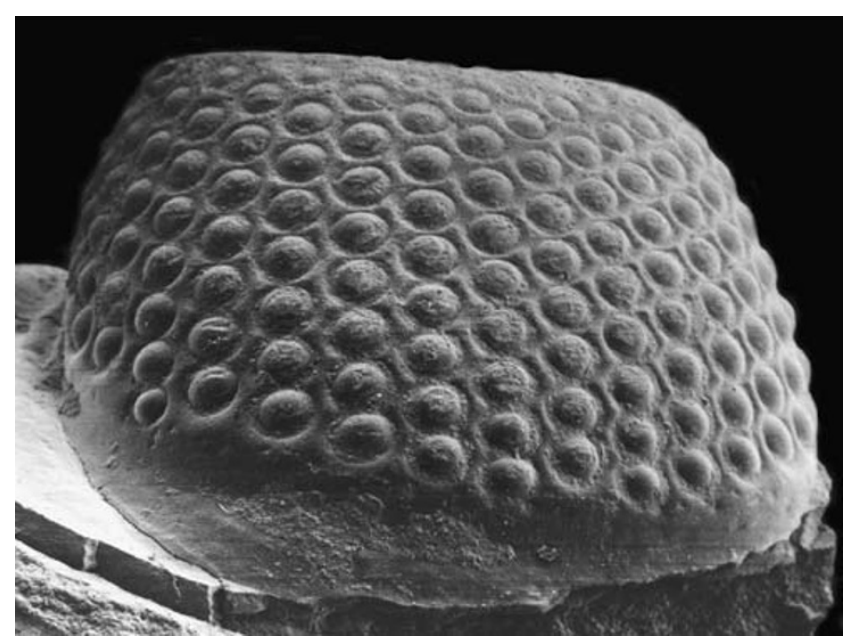

Fig. 2 The fossilized compound eye of the Devonian trilobite species Phacops rana. Note the orderly rows of large lenses. Unlike the compound eyes of modern crustaceans, in these early compound eyes each corneal lens is thought to have served a miniature retina. The overall image would have thus been formed from a mosaic of miniature sub-images 
The trilobite eye illustrated in Fig. 2 displays a more complicated design, called the schizochroal type (Clarke 1889). Here, each corneal lens seen on the surface formed an extended image, using sophisticated optics, and is thought to have served a small retina. Neighboring facets viewed adjacent regions of space, so the visual world was apparently sewn together from dozens of individual mosaic images (Fordyce and Cronin 1989). The design seems to have been ultimately unsuccessful, and the trilobites that had these eyes are long since extinct. Distantly similar eye designs exist today only in a few outlier species of insect larvae and some odd and rare insect parasites, but nothing like schizochroal trilobite eyes ever appeared in crustaceans.

Like the eyes of trilobites, all modern compound eyes are built of repeated, fundamentally similar ommatidial units. A compound eye can be considered to be a collection of dozens to thousands of nearly identical units, each sampling the light of a small region of space for brightness and often for color and polarization as well. The entire visual world is simply the sum of the points sampled by all these ommatidia, much like a digital image is simply a sum of the array of pixels within it. Compound eye images are sometimes portrayed as multiple, repeating views of the same object (much like we see the world through a multifaceted prism), but in fact the overall view is formed in the same way as it is in our retina-each visual unit contributes to one point in the final image. The questions to be considered here are (1) how have these eyes evolved and (2) how are the compound eyes of modern crustaceans related to each other? While general answers to these are clear, the compound eyes we encounter today are so diverse that it is not yet possible to trace all the evolutionary paths they took to get to the present-day designs. To get an appreciation of the diversity that must be explained, we present some examples next.

\section{Crustacean Compound Eye Design}

\section{Sessile Apposition Eyes}

The simplest optical design for a compound eye has each corneal facet devoted to a single photoreceptive rhabdom, making each unit, or ommatidium, a completely independent functional device. The array of ommatidia determines how the visual field is sampled, and each ommatidium is isolated from its neighbors optically and frequently by pigment barriers as well. This forms an apposition compound eye, the most common type found among crustaceans (and arthropods in general; see Figs. 1, 3a, and 4). In the simplest examples of this basic design, the ommatidial array sits on the "head" of the crustacean as a
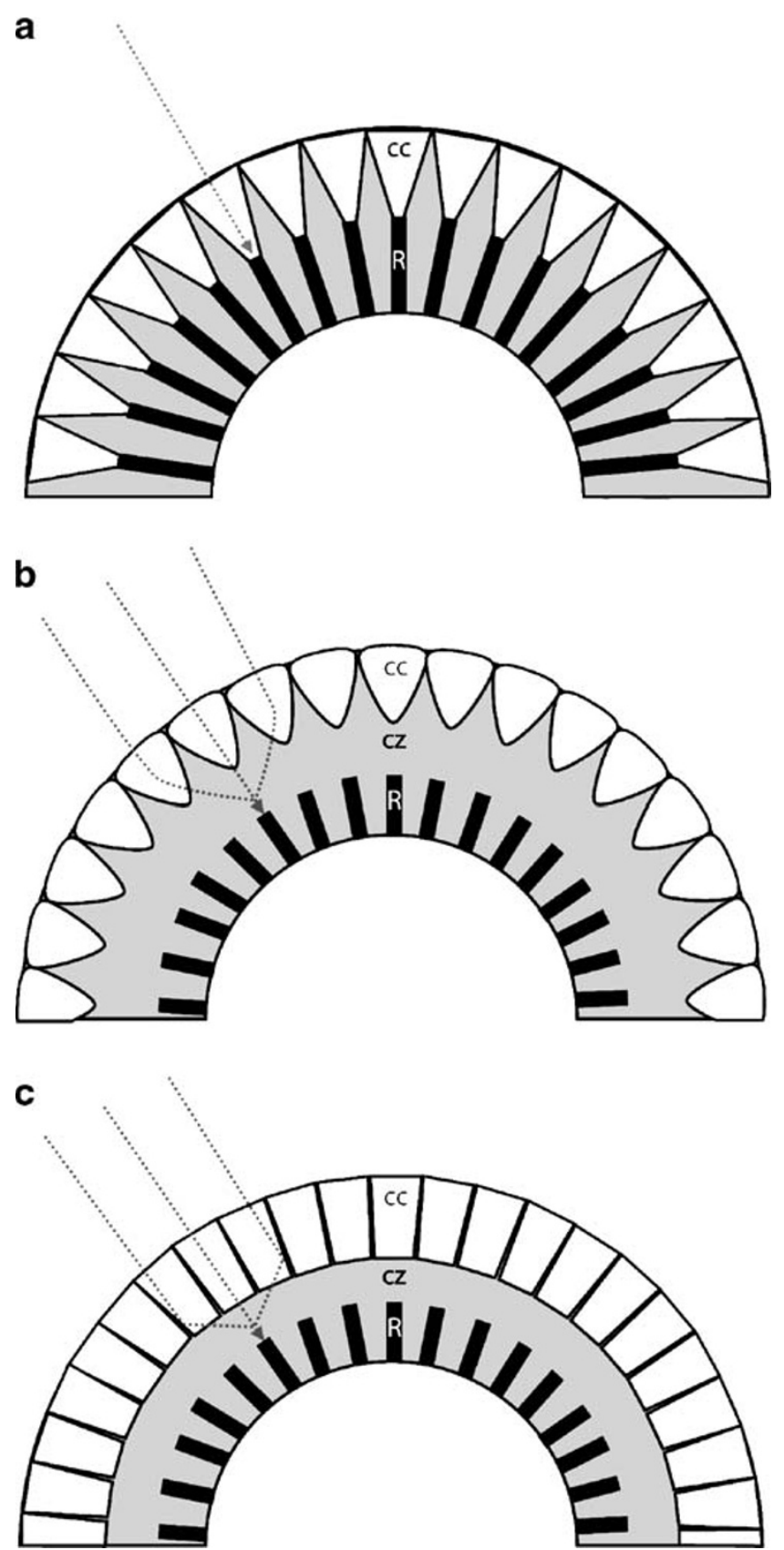

Fig. 3 Schematic diagrams of the three basic compound eye optical designs found in crustaceans. a apposition optics; b refracting superposition optics; c reflecting superposition optics. Dashed grey lines represent typical light paths through the crystalline cones to the rhabdoms. $c c$ crystalline cone, $R$ rhabdom, $c z$ clear zone

raised group of corneal facets. Such eyes can be seen in many isopods and amphipods; for an amphipod example, see Fig. 4a, from Talorchestia longicornis. While the external organization of this eye is rather simple, internally it is quite specialized. Eyes similar to these are found in many small crustaceans, although there are numerous minor taxa where compound eyes are lacking or where they have 

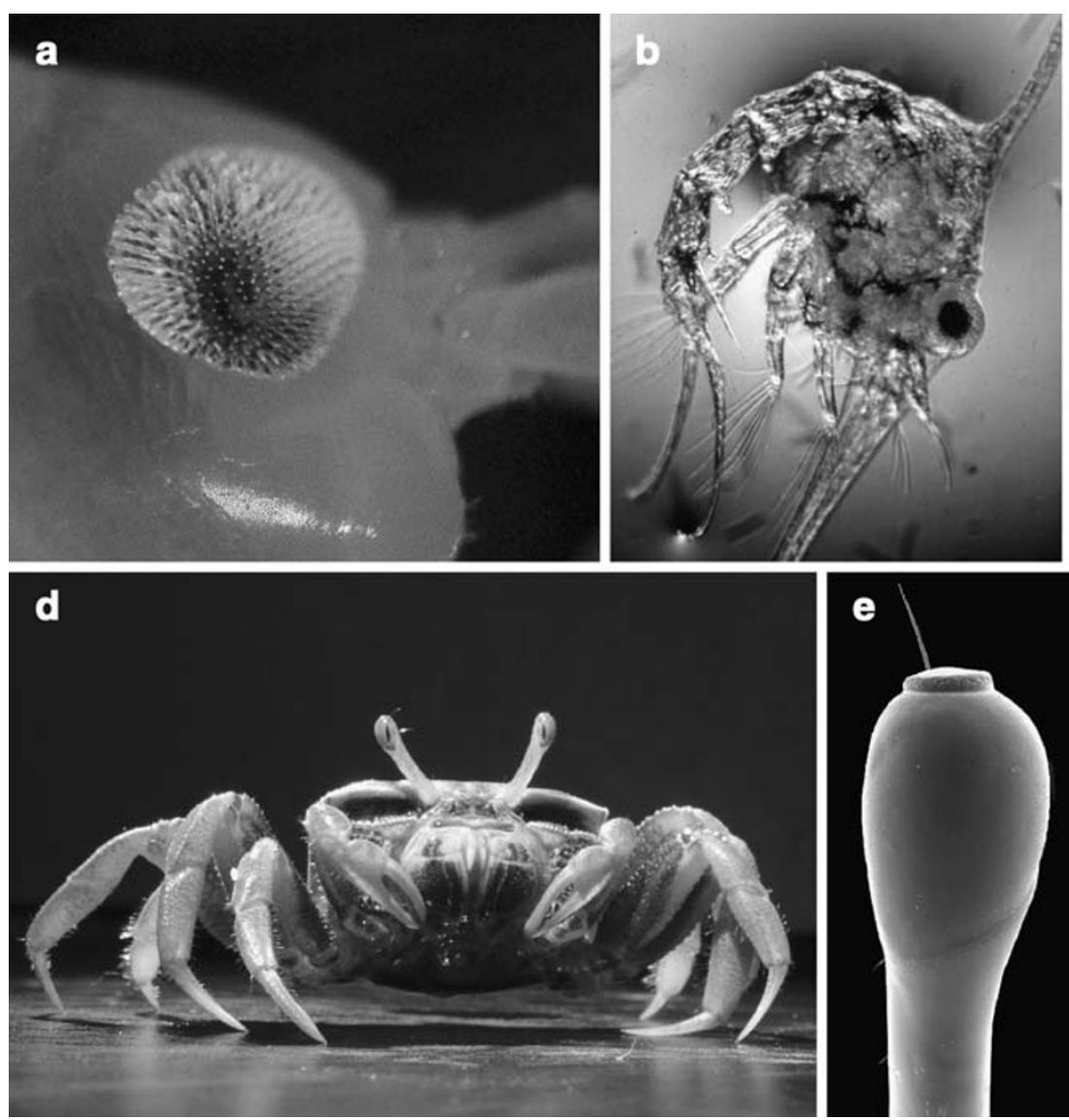

Fig. 4 Examples of apposition or similar types of compound eyes found in modern crustaceans. a The sessile, apposition compound eye of the beach amphipod Talorchestia longicornis. The eye is fixed to the cuticle of the animal, and each corneal facet seen in the photograph sends light to a separate photoreceptive rhabdom (See Fig. 3a for schematic). b A zoeal larval stage of the mud crab Rhithropanopeus harrisii, showing its small and spherical apposition eye. Many crustacean larvae have eyes similar to this. c The double, apposition eyes of the marine hyperiid amphipod Phronima sedentaria. Each eye has two retinas, visible as dark, roughly bean-shaped patches of pigment on each side of the lower part of the head. Photoreceptors in the upper retinas are fed by long fiber optics that lead from corneal facets on the upper, curved surfaces of the animal's

not yet been described (Cronin 1986; Porter and Cronin 2008; see Fig. 1).

The basic apposition design is often modified either subtly or radically in accordance with the lifestyles of particular organisms (see Nilsson 1989). For instance, many larval crustaceans - including all larvae of malacostracanshave transparent apposition eyes, wherein all the pigment is restricted to the compact, central retina (Fig. 4b). Such eyes reduce the visibility of these otherwise transparent plankton. A more profound modification is to collapse the retina into a tiny blob of pigmented receptors and lead light from the very distant corneal facets to it via biological fiber optics. This can produce an eye with excellent visual acuity and rather good sensitivity that is simultaneously almost

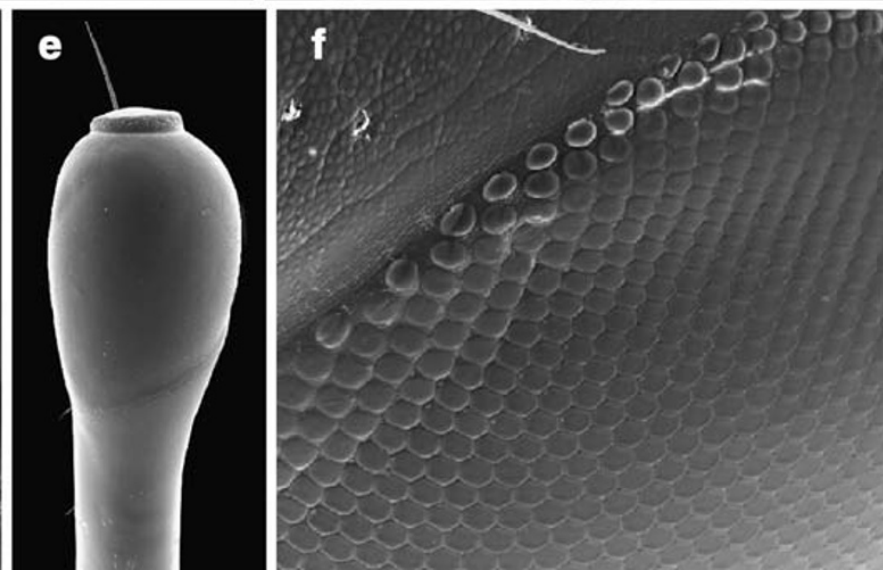

head. These retinas sample only a patch of light overhead, probably searching for objects forming shadows or silhouettes against the dim, down-welling light. The lateral retinas sample space in the remaining parts of the visual field, probably searching for bioluminescent objects. Photograph courtesy of T. Frank, Harbor Branch Oceanographic Institution. $\mathbf{d}-\mathbf{f}$ The stalked apposition eyes of the fiddler crab Uca tangeri, showing how the eyes can be extended above the animal's body for a periscopic and panoramic view. An overall view of a female of this species is illustrated in $\mathbf{d}$ (photograph by F. Fiol), while panels $\mathbf{e}$ and $\mathbf{f}$ show scanning electron micrographs of the ommatidial array and the arrangement of the corneal facets, respectively (photographs by J. Jordão)

invisible, as in the deep-sea hyperiid amphipods (Land 1989; see Fig. 4c). In some small planktonic crustaceans, including the famous "water flea" Daphnia, the two apposition compound eyes are fused side-by-side to form a single spherical organ located under the animal's transparent carapace — a "compound-compound eye"!

\section{Stalked Apposition Eyes}

In most adult malacostracans that have apposition eyes, the eyes no longer sit directly on the carapace and have instead become mounted on stalks. Placing a compound eye on a stalk offers the advantage of an expanded visual field, since the eye now can sit like a periscope away from its owner's 
carapace, and the stalk provides the potential for mobility of the eye as the animal or its visual targets move about. The eyes of fiddler crabs provide superb examples of stalked, "periscopic", moveable eyes (Fig. 4d,e).

Apposition eyes are optically the simplest of compound eye designs, and they tend to be less sensitive than other types. As will be discussed later, superposition eyes offer the potential for greatly enhanced sensitivity. Despite this, animals like isopods and amphipods that live in the deep sea apparently have never evolved superposition compound eyes, and unlike all other deep-sea crustaceans (except possibly some crabs) they continue to see through apposition optics. In some cases, these eyes can achieve outstanding sensitivity, mostly by mating a relatively few large corneal lenses with unusually short focal lengths to fat photoreceptors (Land and Nilsson 2002; Nilsson and Nilsson 1981). To achieve this elevated sensitivity, however, the apposition eye design must decrease acuity, as the large corneal lenses force a reduction in the total number of ommatidia.

While they are optically the simplest of compound eyes, apposition eyes potentially have one huge advantage over the other designs - they can subject different parts of the visual field to different levels of inspection. Because each ommatidium is an individual radiometer for its designated spot in the visual field, the axes of ommatidia can be aligned to be more closely parallel for more intense sampling of a given visual region, or can diverge in regions of lower resolution. As a result, an apposition eye can have one or more patches of ommatidia designed to act like the fovea of a vertebrate eye. These optical regions are usually called "acute zones", because they offer relatively acute vision. For instance, the tall eyes of fiddler crabs (Fig. 4d,e) achieve elevated resolution near the horizon for sampling objects in the flat worlds (e.g. sand flats) they inhabit. Fiddler crab eyes have numerous other specializations for their unusual lives, reviewed by Zeil and Hemmi (2006).

Many other examples of visual field specializations can be found among crustaceans with apposition compound eyes, but the most specialized eyes of all are found in the stomatopods, or mantis shrimp. Mantis shrimps separated from other crustaceans hundreds of millions of years ago, so their evolutionary adaptations have developed over truly deep time. Their eyes are so different from those of all other modern animals, and their early ancestors disappeared so long ago, leaving only rudimentary fossils, that it is difficult now to reconstruct how their present complex forms arose. Mantis shrimp ommatidia are built on a standard crustacean plan, with optics and anatomy much like what is seen in many other species. However, the eye as a whole is divided into three distinct parts: a dorsal region, a ventral region, and an equatorial strip of ommatidia (see Fig. 5). The ommatidia of the dorsal and ventral halves sample extended visual fields like any typical apposition eye, with one unusual aspect: the visual fields of these two halves largely overlap, so many points in the outside world are viewed by both halves of the eye simultaneously. Ommatidia in the equatorial strip, typically arranged into six rows (but sometimes only two or three rows), have visual fields that sample only a planar slice through visual space, placed about midway within the overlapping fields of the surrounding ommatidia. Thus, within this plane, single
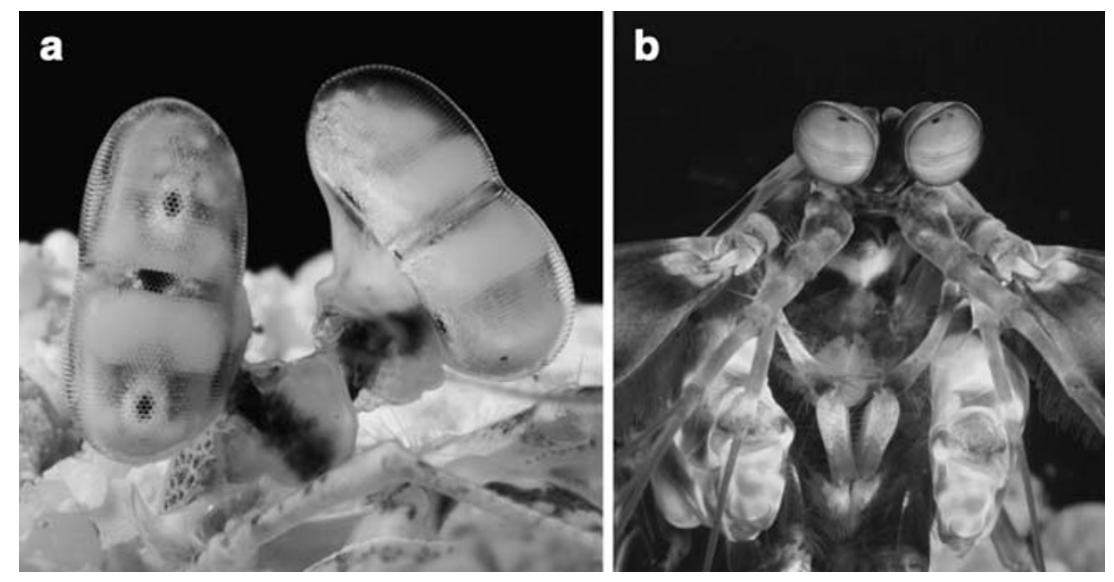

Fig. 5 The very unusual apposition compound eyes of stomatopod crustaceans or mantis shrimps. Panel a shows the tall eye of Lysiosquillina maculata, while Panel b illustrates the more spherically shaped eye of Odontodactylus scyllarus. In both species, the eye is formed from three functional arrays of ommatidia: the dorsal and ventral hemisphere arrays, and the midband array, formed from six parallel rows of ommatidia. See the text for an explanation of how these very unusual eyes function in vision. The tall eye is well adapted for flat-world environments (much like the fiddler crab eye illustrated in Fig. $4 \mathrm{~d}-\mathrm{e}$ ), while the spherical eye is adapted to a more complex visual environment, such as might be found in a rubble field or on a coral reef. Note the dark patches visible on parts of these eyes (particularly the right eye of L. maculata). These indicate the patches of ommatidia that receive light from the direction of the camera, and are called 'pseudopupils'. The presence of three pseudopupils in a single eye shows how the eye views a single point in space from three independent patches of ommatidia. Photographs by R.L. Caldwell 
locations in the external world are seen three times by the same eye.

This twisting and contorting of the eye's surface permits a single eye to estimate the distance to any object in view, as long as it is not too far away. Tall eyes, like those in Fig. 5a, do this with great accuracy, in principle, while round mantis shrimp eyes (Fig. 5b) are probably more limited in the range to which they can carry out accurate rangefinding. The equatorial strips of ommatidia are specialized for high-quality color vision and for analyzing the polarization of light. Since these receptors view the center of the region of overlap, a single point in space can be analyzed simultaneously for form, movement, color, distance, and polarization! No other eye, compound or otherwise, is known to be capable of such high-quality analysis in parallel streams of visual information (Cronin and Marshall 2001). By the way, the ability of mantis shrimp eyes to analyze light's polarization might seem exotic, but in fact, most arthropods have this ability.

Stomatopods have taken the apposition design just about to its limit, and as might be expected, their specializations have an evolutionary cost. Because only a tiny slice of the field of view can be analyzed so thoroughly, due to the triply overlapping design, the eye must constantly move to integrate all possible aspects of visual information. This, of course, costs the animal time and must occasionally cause the mantis shrimp to miss seeing something important, especially if the object is only briefly in view.

\section{Superposition Compound Eyes}

Unlike apposition eyes, in which each ommatidium is an independent visual unit, superposition compound eyes have ommatidia where the optics function cooperatively. Groups of corneal lenses focus light originating from a single point in space onto a single rhabdom. As in the apposition type, each photoreceptor still views an assigned spatial location, and thus the density of sampling of the visual field remains a function of the number of ommatidia. But each rhabdom now receives light through numerous different facets (see Fig. 3b,c), making for a much brighter image than is normally possible using the apposition design. Superposition eyes have repeatedly evolved in both insects and in crustaceans and are thus taxonomically widespread (Land and Nilsson 2002, Nilsson 1989; see Fig. 1).

Forming a superposition image is not optically straightforward. Single optical units in the cornea (typically joined to crystalline cones) must redirect light, not only the rays that are axially aligned with the rhabdom (the only responsibility of the lenses of an apposition eye), but also other rays entering the facet over a wide range of spatial angles. All these rays must be correctly sent to rhabdoms making up a significant subset of the photoreceptor array. If this is done using refractive lenses (Fig. 3b), by far the most common optical devices used in living (and artificial) systems, the image that is formed must be "erect;" in other words, the top of the image should correspond to the top of the focused object, right must be to the right, and so on. This is the precise opposite of an image formed by a typical lens, as in a human eye, which is always inverted and reversed.

This paradoxical situation was resolved through the insights of Sigmund Exner, who published the first modern treatise on compound eye physiology over a century ago (1891; a full English translation by R.C. Hardie was released in 1989). The erect image is produced because the lens-cone unit has an internal gradient of refractive index that is radially symmetrical around its long axis. The resulting bullet-shaped optic is called a "lens cylinder." Superposition eyes have another optical requirement; they need to leave space inside the eye's volume to allow the correctly formed rays exiting the erecting lenses to have the opportunity to join with rays from other lenses arising from the same spatial location. Thus, a diagnostic feature of the anatomy of the superposition type is the presence of a gap, or space, between the superficial optical layer and the deeper array of photoreceptive rhabdoms; this is called the "clear zone" (see Fig. 3b,c). Apposition eyes lack this zone because the rhabdoms invariably abut the terminations of the image-forming optics (or, in the cases of apposition eyes with fiber optics, the terminations of the light guides).

While they certainly can produce brighter retinal illumination, superposition eyes do not offer the flexibility of spatial sampling available to apposition eyes. Because the various lenses must work together, the eye cannot be distorted or reshaped easily to form acute zones, overlapping visual fields, or other types of specializations that are frequently encountered in apposition eyes. However, the eye is not without recourse to some specialization. Eyes of euphausiids (krill) and mysids form superposition images by refraction using lens cylinders, and in shallow-living species these eyes are usually spherical, seeing about equally well in all directions. In deep-sea species, however, the superposition eye is very often formed into an hourglass or dumbbell shape (Fig. 6). The upper half is expanded and flattened, looking upward to produce a greatly enhanced superposition image of the dim light filtering down from the distant surface of the ocean. This is joined at the equator with a more normal-looking lower half having a nearly spherical field of view, which is presumably devoted to searching for bioluminescent flashes in other directions. Another very unusually modified superposition eye exists in a mysid, Dioptromysis paucinispinosa, described by Nilsson and Modlin (1994). Here, most of the ommatidia are arranged in the conventional spherical array, but a single corneal facet near the margin of the eye is hugely enlarged, 


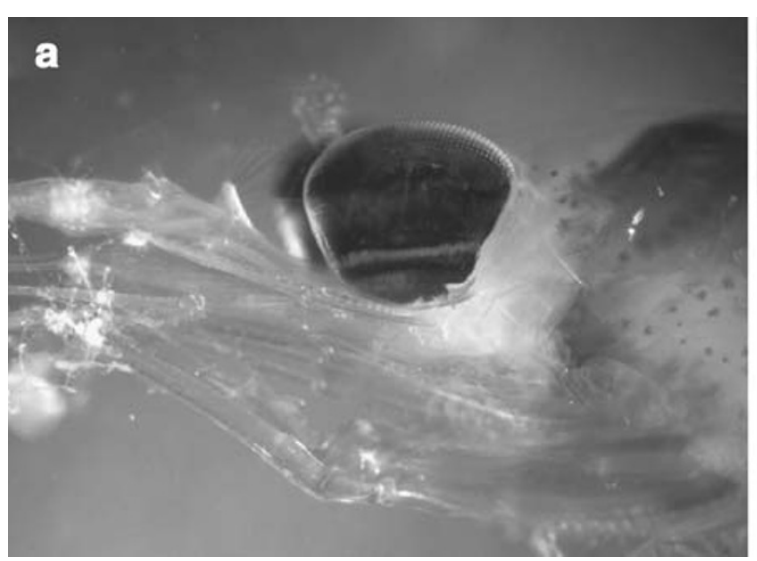

Fig. 6 Double, superposition compound eyes found in some deep-sea euphausiid crustaceans (better known as 'krill'). The euphausiids are one of the few crustacean groups containing refracting superposition eyes, with optics similar to the schematic in Fig. 2 b. The eyes shown here are rather unusual in that the dorsal half is arrayed so as to inspect just the downwelling light arriving from the ocean above, while the ventral half forms a nearly complete spherical array sampling in all other directions. These eyes are thus superposition analogues of the

and is matched to a local retina of over 100 photoreceptors. Apparently the optics of most of the original ommatidia have been lost here, leaving only a single optical unit of cornea and crystalline cone, forming a gigantic lens cylinder. Because erecting optics are present in the relatively huge corneal facet, the retina here sees an erect image which is greatly magnified compared to what is seen by the rest of the ommatidial array. It is thought that this miniature telescope in each eye is used to search for food or mates, but behavioral observations are thus far absent.

The refracting superposition design is seen in insects as well as crustaceans, but crustaceans have evolved two other superposition designs rarely or never occurring anywhere else. Both of these use reflection of light rays either in part or wholly to form the superposition image. The more widespread of these designs is based entirely on reflection for image formation and is appropriately called the reflecting superposition eye (Fig. 3c). This eye type is known only for decapod crustaceans, but it is fairly widespread among them, existing in almost every decapod order (Fig. 1; Porter and Cronin 2008).

Reflecting superposition seems out of the ordinary, but in fact the principle is simple and straightforward. Recall that formation of a superposition image requires that light rays be bent back towards the appropriate receptor. Essentially, this is what a mirror does; it reflects rays back towards their sources. If two mirrors are mounted at $90^{\circ}$ to each other, they will form a completely upright image, and if four mirrors are joined to form a long box with a square opening, any light ray entering the box from one end will be reflected out the other end at an angle essentially identical to that produced by refracting superposition optics. Notice that the light rays in Fig. 3c follow very similar paths to those in Fig. $3 \mathrm{~b}$, even though the optical mechanisms are quite distinct. Biological mirrors are actually quite common and produce the eyeshine of many animals, from shrimps and spiders to cats. They are usually built of crystals of guanine or other reflective biological material, although many other means of reflecting light also exist; see Land and Nilsson (2002) for other examples and a very readable account of mirrors in eyes. The trick that makes reflecting superposition eyes work is the presence of square corners in the corneal facets and in the mirror boxes below them. Thus, an immediate indication that a crustacean eye is of the reflecting superposition type is the presence of square facets (see Fig. 7 for an example from a decapod shrimp). The square facets in the eyes of lobsters are large enough to be seen (barely) without the aid of a microscope.

The third means of producing a superposition image was worked out by Dan-Eric Nilsson of the University of Lund only 20 years ago (Nilsson 1988), making it the most recently discovered biological, image-producing system. Nilsson terms this "parabolic superposition", because the sides of the mirrors, formed from the crystalline cones, are shaped in the form of a parabola as seen in longitudinal section. The eye as a whole looks much like a refracting superposition eye, having hexagonal facets and roughly bullet-shaped crystalline cones (like those in Fig. 3b), but there are no lens cylinders present. The principle is complicated, and Nilsson's original paper (or Land and Nilsson 2002) should be consulted for a full explanation, but the basic idea is that the corneal lenses focus light refractively, and the beams entering the crystalline cone are 


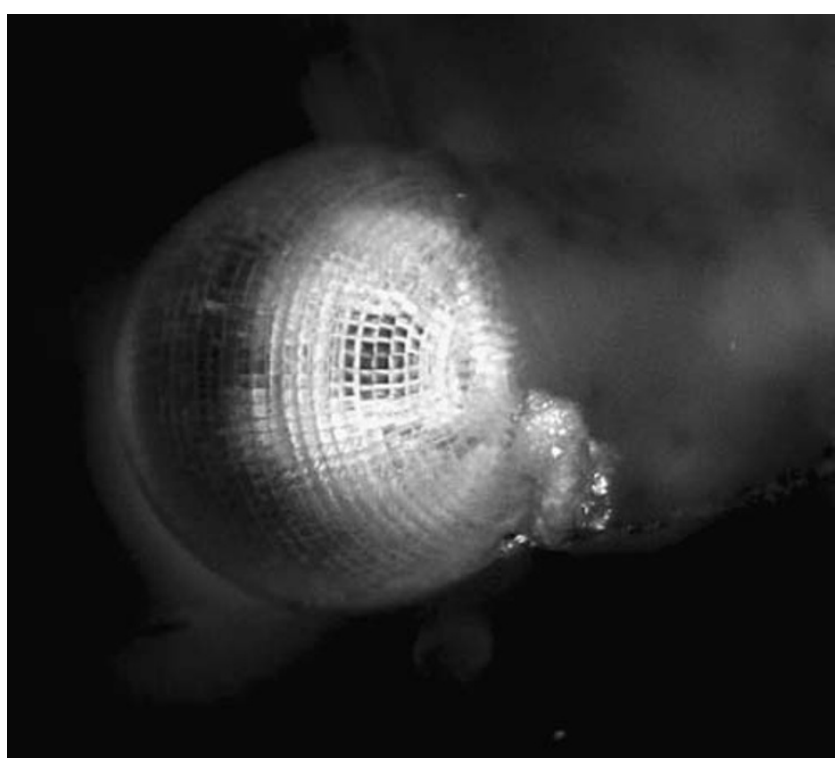

Fig. 7 The reflecting superposition eye of the grass shrimp, Palaemonetes pugio. Note the square facets easily visible on the surface, which are nearly diagnostic of this optical design (see Fig. 3c for a schematic of reflecting optics)

redirected by the parabolic mirrors in the appropriate directions to form a nearly perfect erect superposition image. The design as a whole incorporates elements of both refracting and reflecting superposition optics (as well as some of the features of apposition eyes) and seems to have been an intermediate in the evolution of eyes in some animals whose close ancestors use other optical principles.

\section{Evolution of Compound Eyes}

It's obvious from the preceding discussion that crustacean compound eyes come in a bewilderingly diverse set of designs. In addition, the optical principles of one type may not be easily compatible with those of a different design, so it is often difficult to envision the intermediates that link the fully developed eye designs encountered in today's crustaceans. For example, it is not difficult to conceive how a sessile compound eye can be altered into a stalked eye of the same basic design (say, apposition), but it is more problematic to see the evolutionary route from apposition to reflecting superposition. Making the problem more complicated, optics and internal structures of fossil compound eyes are usually difficult - if not impossible - to work out (with trilobites, as in Fig. 2, being the exception), so reconstructing the operating principles of early compound eyes is rarely practical.

The best evidence available today to help us understand crustacean compound-eye evolution comes from their development. The large and often optically complex eyes of adult stomatopods, euphausiids, and decapods almost all originate from the much simpler apposition compound eyes of their larvae, as in the transparent apposition eye of the crab larva seen in Fig. 4b (the only exceptions are species that show direct development, like crayfish). These eyes are optically simple, while being at the same time well designed for the needs of larvae. They are mostly transparent, having a compact retina, while providing panoramic coverage for orientation and for detection of oncoming predators (and perhaps for locating food as well). The apposition eye continues to function in many adult crustaceans, like some crabs, which carry the larval plan through metamorphosis, remodeling it by enlarging the eye and adding pigment and support.

While the developmental path from a transparent apposition eye in the larva to any of the types of superposition eye seems cryptic, it turns out that larval eyes already have structural features that are compatible with either apposition, refracting superposition, or reflecting superposition optics in adults. Nilsson (1983) noted that crustacean larval eyes, despite their fundamental apposition optics, form superposition rays within the crystalline cones that are not used for image formation but that are readily adaptable for use in the adult eye. Thus, the roots of both reflecting and refracting superposition eyes reside in the larvae. The opposite transition can occur as well, accounting for the appearance of apposition compound eyes in groups of crustaceans that normally possess only superposition eyes. For instance, while decapod shrimps in general have reflecting superposition eyes as adults (e.g. Fig. 7), adult shrimps of the family Spongicolidae may possess apposition eyes produced by neoteny (Gaten 2007). Interestingly, mysids brood their young, so the juveniles are never planktonic; yet developing mysids also have apposition compound eyes that transform to refracting superposition eyes in the adults (Nilsson et al. 1986). Richter (2002) has argued convincingly that paedomorphosis can account for the appearances of unexpected compound eye types in adults of several crustacean taxa, and he has presented a revised view of compound eye evolution incorporating this premise.

Paradoxically, there is one group of crustaceans that does have apposition eyes both as larvae and as adults, but where the larval retina is completely discarded at metamorphosis. This is the stomatopod crustaceans. As noted above, stomatopods (mantis shrimps) have very unusual eyes with multiple overlapping visual fields and subgroups of receptors specialized for different visual tasks. Some of these specializations have involved fairly radical reorganization of photoreceptors, and oddly enough (and apparently uniquely for crustaceans) these receptors are constructed anew at the time of metamorphosis from larva to juvenile (see Cronin et al. 1995; Cronin and Jinks 2001). A complete, new retina forms alongside the larval one at this 
time, and in the newly molted juvenile a remnant of the larval retina remains for a while but ultimately disappears (see Cronin and Jinks 2001, for a brief review and a list of references).

The reason for this radical departure from crustacean orthodoxy apparently can be traced to the extremely unusual structures in the specialized rhabdoms that stomatopods use to analyze the spectral content (or color) of light. These photoreceptors are arranged into stacks, or tiers, such that light is sequentially absorbed in different parts of the spectrum as it passes through the entire length of the rhabdom (see Cronin and Marshall 2004, for a review). The unusual design is clearly a modification of the most common crustacean plan, where each rhabdom is formed from the fusion of typically eight receptor cells, called "retinular cells." In many crustaceans, a short-wavelengthsensitive photoreceptor type, called an R8 (meaning "retinular cell number 8") is placed on top of a group of the other seven cells, R1-7 (see Fig. 8, "Step 1"). Rhabdoms like this are found in all parts of mantis shrimp eyes that are not involved with color vision, but ommatidia specialized to analyze color break up the R1-7 group into two subgroups placed on top of each other, as in Fig. 8, "Step 2." This produces a three-tiered rhabdom, with each tier sampling a different part of the spectrum, and these rhabdoms are devoted to shorter-wavelength regions of light (violet through green). Apparently to improve the discrimination of longer-wavelength light (yellow through red) by stomatopods, evolution has added colored filters between the successive tiers of some classes of rhabdoms. In more primitive species, one filter is added at the junction of the R8 cell and the divided group of R1-7 (Fig. 8, "Step 3"). The species of mantis shrimps with the most complex eyes have yet another filter at the junction between the two subgroups of R1-7, as suggested by Fig. 8, "Step 4." This progression of complexity thus parallels stomatopod evolution in general and ultimately leads to an extremely complicated but highly functional compound eye.

A discussion of crustacean eye evolution would not be complete without a brief reference to eyes that have evolved in the opposite direction to those of mantis shrimps, towards extreme simplicity. Crustaceans inhabiting the deepest regions of the ocean, and in particular the inhabitants of the hydrothermal vents of the mid-Atlantic Ridge or the Galapagos Rift, have often done away with optics and separate ommatidia altogether, forming the retina into a thick sheet of photosensitive material with no remaining sign of its origin. A good example of this reduction is seen in the hydrothermal vent shrimps (Van Dover et al 1989; Jinks et al. 1998), where in some species the retina has left the eyestalks entirely and migrated onto the dorsal region of the carapace. In the brachyuran vent crab Bythograea thermydron, larvae and postlarvae have conventional apposition eyes, but the eye regresses in the adults to form a nearly featureless mass of tissue containing the visual pigment (Jinks et al. 2002). These eyes are still organs of sight, but they are no longer able to form images and instead are thought to act as anti-boil devices, responsible for sighting the dim, red light emitted from the hot water issuing from the vents.

\section{Molecular Evolution of Vision in Crustacea}

As we have illustrated in the previous sections, a considerable amount of research has been devoted to the
Fig. 8 A series of schematic diagrams to suggest the evolution of complex photoreceptor sets in stomatopod crustaceans (mantis shrimp). The receptor arrangement illustrated in 'Step 1' is typical of that found in many crustaceans, as well as in some ommatidia of mantis shrimps. The other arrangements are found only in the stomatopods. See the text for an explanation of the figure

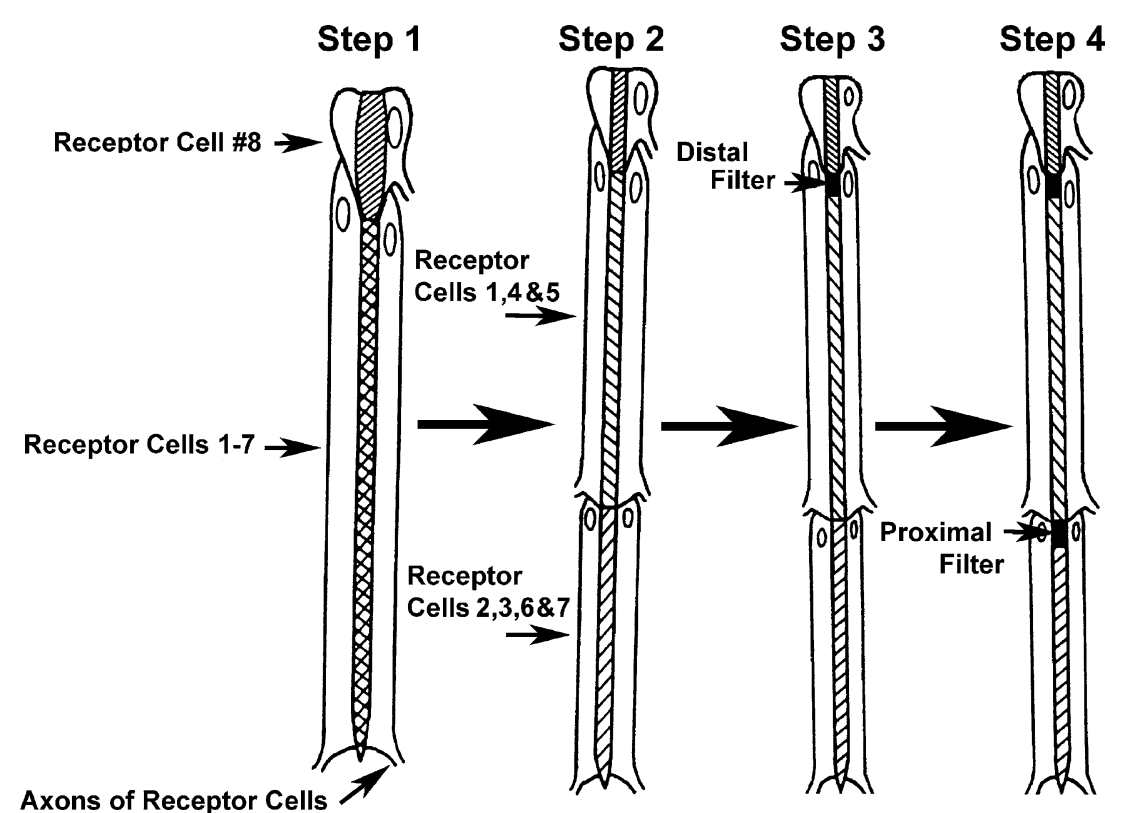


evolution of crustacean visual system morphologies and structures (also see reviews by Cronin 1986, 2005). The optical structures and photoreceptors within the eye are all designed for the efficient capture of the light available within a given habitat, from the infrared of the deep-sea vents to the full spectrum available to shallow coral reef dwellers. At the foundation of any visual system, however, are the molecules responsible for absorbing photons of light and converting this photic energy into a cellular signal. Regardless of the overlying structure of the eye, the underlying light-capturing molecule is always a visual pigment composed of two parts: a chromophore (vitamin A derivative) bound to an integral membrane protein (opsin). Visual pigments are characterized by the wavelength of light at which they absorb maximally. Different visual pigments can be 'tuned' to different wavelengths of light by the interaction between the chromophore and specific amino acids within the opsin protein. In the typical crustacean eye design, the photoreceptor cells R1-7 have a spectral sensitivity in the blue-green range (around $500 \mathrm{~nm}$ ) while the R8 cell has a spectral sensitivity in the violet to ultraviolet range (see Fig. 8 'Step 1' for photoreceptor schematic); these differences in sensitivity imply that each photoreceptor type expresses a different opsin, forming a unique visual pigment. However, very few studies have investigated the molecular evolution of crustacean visual systems to explore how the evolution of the genes responsible for vision (e.g. opsins) matches the evolution of the structures in which they are found.

If we look at the evolution of the available crustacean opsin genes relative to the opsins known from the other major arthropod groups (e.g. insects and arachnids), several important evolutionary patterns can be observed (Fig. 9). With the interesting exception of opsin sequences from brachyuran crabs, all of the crustacean opsins form a single sequence cluster. This main crustacean cluster is most closely related to opsin sequences from insects (Hexapoda) and chelicerates (spiders and allies) that form visual pigments sensitive to longer wavelengths of light (generally greater than $480 \mathrm{~nm}$ ). So far, although photoreceptors sensitive to violet and ultraviolet light have been physiologically characterized in crustaceans (generally the R8 cells), no full-length sequences of these shorter wavelength opsins have been published yet. Incomplete opsin sequences, thought to be from photopigments sensitive to blue wavelengths of light, have been isolated from ostracods (Oakley and Huber 2004). Although the shorter lengths of these sequences prevented us from including them in our phylogenetic analyses, their existence (in conjunction with the placement of the main clade of crustacean opsins) suggests that arthropod visual systems evolved from an ancestor containing at least two visual pigments - one sensitive to longer, blue-green wavelengths of light and another sensitive to shorter, violet to ultraviolet wavelengths of light.

The only opsin sequences that don't fall within the main crustacean group are those from brachyuran crabs. Although the eyes of brachyuran crabs are structurally similar to those of other, closely related crustacean species, the opsins contained within their photoreceptors are quite unique relative to other characterized crustacean and arthropod sequences. Furthermore, the species that has been described most completely, Hemigrapsus sanguineus, expresses two distinct opsin sequences within the main photoreceptor (indicated in Fig. 9 by triangles). The expression of two middle-wavelength opsin sequences has also been shown in a deep-sea species of lophogastrid, Gnathophausia ingens. Because the $G$. ingens visual system lacks R8 cells (Frank et al. 2008), it is assumed that both of these opsins also are expressed in the main photoreceptor (Fig. 9, indicated by squares). Similarly, Penaeus monodon also appears to express two very different opsin sequences (Fig. 9, indicated by stars), although where these two opsins are expressed is unknown. Unlike insects, which appear to have a fairly well defined set of expressed opsins, with each photoreceptor cell generally expressing a single opsin gene, crustacean opsins appear to be more 'promiscuous'. There is mounting evidence, including the cases presented above, and another even more extreme case in ostracods (Oakley and Huber 2004), that the expression of multiple opsin genes in single photoreceptors is common in crustaceans.

\section{Summary}

Within this review we have illustrated how the diversity of anatomical forms that exists throughout the Crustacea is matched by the optical, structural, and molecular diversity contained within crustacean visual systems. Although our understanding of the evolution of this diversity is hampered by our ever-shifting view of crustacean phylogenetic relationships, several key ideas have emerged. While there are a number of crustacean groups with simple eyes, or no eyes at all, most crustacean lineages contain compound eyes with apposition optics. Even those species with superposition eyes as adults have apposition eyes in larval forms, indicating that apposition eyes arose early within the crustaceans. Superposition eyes, then, represent a more derived eye type that appears in three different crustacean groups - the decapods, euphausiids, and mysids - suggesting at least two independent origins (Fig. 1). Similarly, crustaceans have put their eyes onto stalks multiple independent times, suggesting that this is a visually advantageous arrangement.

It is also apparent from looking at the distribution of compound eye types within the crustacean phylogeny that 


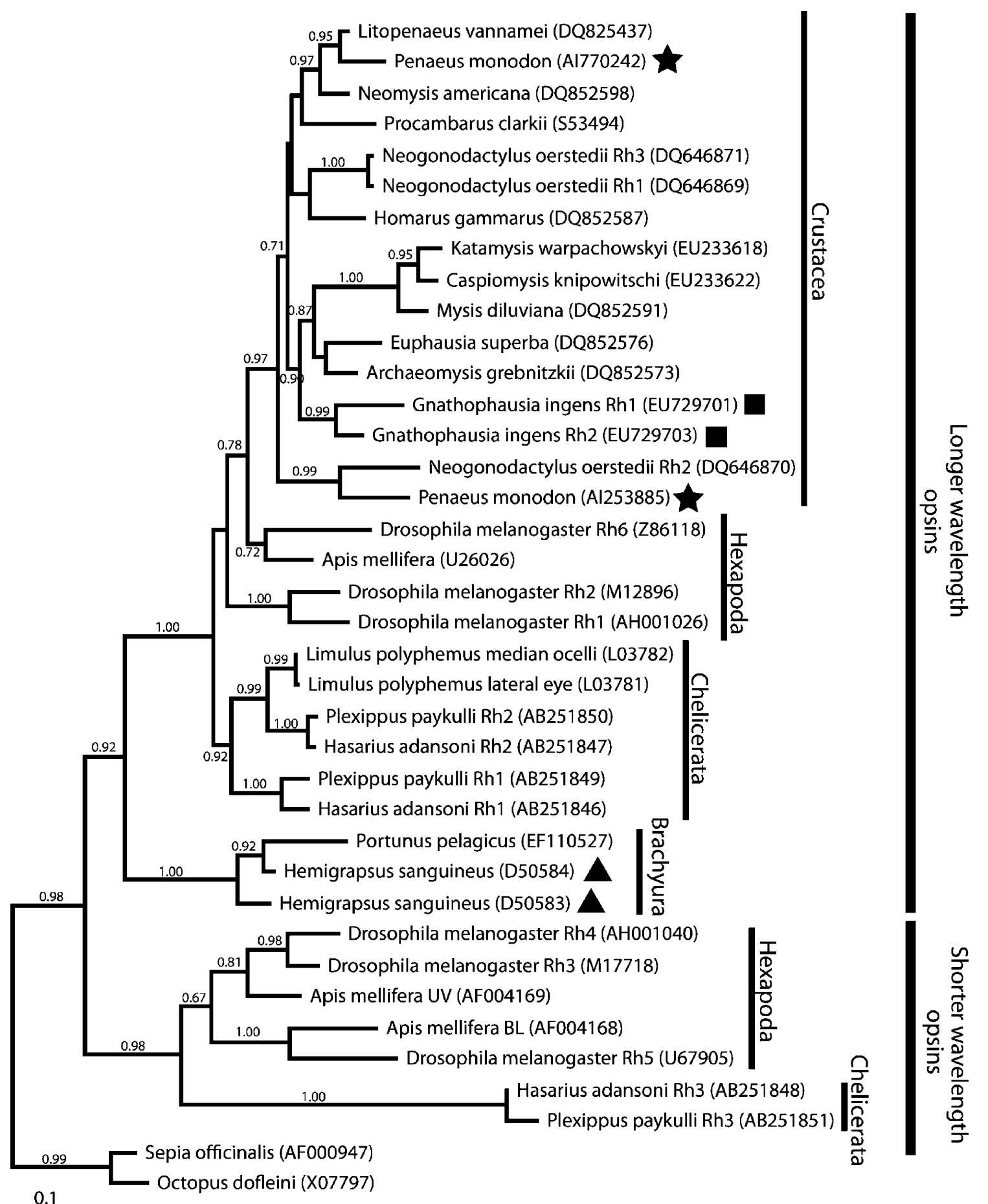

Fig. 9 Phylogeny of publicly available crustacean and selected invertebrate opsins based on maximum likelihood analyses of amino acid residues. The phylogeny was reconstructed using PHYML (Guindon and Gascuel 2003) and rooted (not shown) using bovine rhodopsin (NC 007320), chicken pinopsin (U15762), and human melatonin receptor 1A (NM_005958) and GPCR52 (NM_005684). Accession numbers are provided in parentheses behind each species name and the numbers above each branch indicate the bootstrap proportion from 100 replicates (values less than 0.70 not shown). The taxonomic groups of major clusters of opsin sequences are indicated. The division between opsins forming visual pigments with sensitivity to longer wavelengths of light (blue-green to red) versus shorter wavelengths of light (ultraviolet to blue) has also been designated. Species where more than one opsin sequence has been isolated are indicated by symbols: stars $=$ Penaeus monodon, squares $=$ Gnathophausia ingens, and triangles $=$ Hemigrapsus sanguineus 
most of the diversity of optical designs is found in the larger species. In particular, the decapods (crabs, shrimps, and lobsters) exhibit all known optical types of crustacean eyes, including the reflecting and parabolic superposition types not found in any other groups (Fig. 1). This diversity in part reflects the large number of species within the decapods as well as their ecological variability. However, this is not a complete explanation for the presence of unique optical designs only within the decapods, which remains a puzzle to be solved.

Finally, there are many instances of convergence, where multiple lineages have gained or lost traits independently. As just mentioned, it is likely (although still open for debate) that refracting superposition optics and the stalkedeye arrangement both evolved independently multiple times. Similarly, a number of lineages have independently lost eyes altogether. Although based on different underlying optical types, the eye designs of the hyperiid amphipod Phronima (Fig. 4c) and the double-eyed euphausiids (Fig. 6) and mysids (Gaten et al. 2002) - which occupy similar ecological environments in the deep sea-illustrate a functional convergence imposed by similar evolutionary pressures on the visual system to sample in different directions in space for different purposes.

Significantly, the phylogenetic relationships among crustaceans are still contentious, particularly in comparison with the evolutionary relationships that are well documented among most other modern animals. A thorough understanding of crustacean visual system evolution will become possible only with the establishment of a cohesive, well-supported crustacean phylogeny. Correspondingly, as we move towards this goal, a consideration of how compound eyes arose and diversified will be an important facet for improving our understanding of crustacean evolution. The examples we have presented here represent many of the bewildering types of eyes found in the crustaceans, illustrating how fundamentally different optical principles can be derived from each other and highlighting both our current understanding of the evolution of this visual diversity and the mysteries that still remain to be uncovered.

Acknowledgements This work was supported by grants from NSF (IOS-0721608) and AFOSR (02NL253).

\section{References}

Clarke JM. The structure and development of the visual area in the trilobite Phacops rana. Green. J Morphol 1889;2:253-70. doi:10.1002/jmor.1050020203.

Cronin TW. Optical design and evolutionary adaptation in crustacean compound eyes. J Crustac Biol 1986;6:1-23. doi:10.2307/ 1547926.
Cronin TW. Invertebrate vision in water. In: Warrant E, Nilsson D-E, editors. Invertebrate Vision. Cambridge, UK: Cambridge University Press; 2005. p. 211-49.

Cronin TW, Jinks RN. Ontogeny of vision in marine crustaceans. Am Zool 2001;41:1098-107. doi:10.1668/0003-1569(2001)041[1098: OOVIMC]2.0.CO;2.

Cronin TW, Marshall NJ. Parallel processing and image analysis in the eyes of mantis shrimps. Biol Bull 2001;200:177-83. doi:10. 2307/1543312.

Cronin TW, Marshall NJ. The visual world of mantis shrimps. In: Prete FR, editor. Complex Worlds from Simpler Nervous Systems. Cambridge, MA, USA: MIT Press; 2004. p. 239-68.

Cronin TW, Marshall NJ, Caldwell RL, Pales D. Compound eyes and ocular pigments of crustacean larvae (Stomatopoda and Decapoda, Brachyura). Mar Freshwat Behav Physiol 1995;26:219-31.

Exner S. The Physiology of the Compound Eyes of Insects and Crustaceans. Translated from the German by R.C. Hardie (1989), republished by Springer, Heidelberg; 1891.

Fordyce D, Cronin TW. Comparison of fossilized schizochroal compound eyes of phacopid trilobites with eyes of modern marine crustaceans and other arthropods. J Crustac Biol 1989;9: 554-69. doi:10.2307/1548587.

Fordyce D, Cronin TW. Trilobite vision: a comparison of schizochroal and holochroal eyes with the compound eyes of modern arthropods. Paleobiology 1993;19:288-303.

Frank TM, Porter ML, Cronin TW. Spectral sensitivity, visual pigments and screening pigments in two life history stages of the ontogenetic migrator Gnathophausia ingens. J Mar Biol Assoc UK. 2008; In press. doi:10.107/S0025315408002440.

Gaten E. Apposition compound eyes of Spongicoloides koehleri (Crustacea: Spongicolidae) are derived by neoteny. J Mar Biol Assoc U K 2007;87:483-6. doi:10.1017/S002531540705597X.

Gaten E, Herring PJ, Shelton PMJ. Eye morphology and optics of the double-eyed mysid Euchaetomera typica. Acta Zool 2002;83: 221-30. doi:10.1046/j.1463-6395.2002.00116.x.

Guindon S, Gascuel O. A simple, fast, and accurate algorithm to estimate large phylogenies by maximum likelihood. Syst Biol 2003;52:696-704. doi:10.1080/10635150390235520.

Jinks RN, Battelle BA, Herzog ED, Kass L, Renninger GH, Chamberlain SC. Sensory adaptations in hydrothermal vent shrimps from the Mid-Atlantic Ridge. Cah Biol Mar 1998;39: 309-12.

Jinks RN, Markley TL, Taylor EE, Perovich G, Dittel AI, Epifanio $\mathrm{CE}$, et al. Adaptive visual metamorphosis in a deep-sea hydrothermal vent crab. Nature 2002;420:68-70. doi:10.1038/ nature01144.

Land MF. Crustacea. In: Ali MA, editor. Photoreception and Vision in Invertebrates. New York: Plenum Press; 1984. p. 401-38.

Land MF. The eyes of hyperiid amphipods: relations of optical structure to depth. J Comp Physiol [A] 1989;164:751-62. doi:10. 1007/BF00616747.

Land MF, Nilsson DE. Animal Eyes. Oxford, UK: Oxford University Press; ; 2002.

Martin JW, Davis GE. An updated classification of the recent Crustacea. Science Series, 39. Natural History Museum of Los Angeles County: Los Angeles, CA (USA); 2001. vii, 123 pp.

Nilsson DE. Evolutionary links between apposition and superposition optics in crustacean eyes. Nature 1983;302:818-21. doi:10.1038/ $302818 \mathrm{a} 0$.

Nilsson DE. A new type of imaging optics in compound eyes. Nature 1988;332:76-8. doi:10.1038/332076a0.

Nilsson DE. Optics and evolution of the compound eye. In: Hardie RC, Stavenga DG, editors. Facets of Vision. Heidelberg, Germany: Springer; 1989. p. 30-73.

Nilsson DE, Hallberg E, Elofsson R. The ontogenetic development of refracting superposition eyes in crustaceans: transformation of 
optical design. Tissue Cell 1986;18:509-19. doi:10.1016/00408166(86)90017-0.

Nilsson DE, Modlin RF. A mysid shrimp carrying a pair of binoculars. J Exp Biol 1994;189:213-36.

Nilsson DE, Nilsson HL. A crustacean compound eye adapted to low light intensities. J Comp Physiol [A] 1981;143:503-10. doi:10. 1007/BF00609917.

Oakley TH, Huber DR. Differential expression of duplicated opsin genes in two eye types of ostracod crustaceans. J Mol Evol 2004;59:239-49. doi:10.1007/s00239-004-2618-7.

Porter ML, Cronin TW. A shrimp's eye view of evolution: how useful are visual characters in decapod phylogenetics? Crustac Issues. 2008; In press.
Richter S. Evolution of optical design in the Malacostraca (Crustacea). In: Wiese K, editor. The Crustacean Nervous System. Heidelberg, Germany: Springer; 2002. p. 512-24.

Schram FR, Koenemann S. Are the crustaceans monophyletic? In: Cracraft J, Donoghue MJ, editors. Assembling the Tree of Life. Oxford, UK: Oxford University Press; 2004. p. 319 29.

Van Dover CL, Szuts EZ, Chamberlain SC, Cann JR. A novel eye in "eyeless" shrimp from hydrothermal vents of the Mid-Atlantic Ridge. Nature 1989;337:458-60. doi:10.1038/337458a0.

Zeil J, Hemmi JM. The visual ecology of fiddler crabs. J Comp Physiol [A] 2006;192:1-25. doi:10.1007/s00359-0050048-7. 